\title{
Contact Precautions for Methicillin-Resistant Staphylococcus aureus: Are They Still Valuable?
}

\author{
Jennifer Brown
}

Published online: 30 September 2014

(C) Springer Science+Business Media New York 2014

\begin{abstract}
Guidelines from the US Centers for Disease Control and Prevention and the Healthcare Infection Control Practices Advisory Committee recommend the use of contact precautions to prevent the transmission of methicillin-resistant Staphylococcus aureus in acute-care facilities. The merits of these recommendations are debated because it is not clear that contact precautions are essential for the control of this drug-resistant bacterium. Also, contact precautions are resource-intensive and may be detrimental to patient well-being. Recent studies have demonstrated that horizontal measures, such as improved hand hygiene compliance and chlorhexidine body washing, may be effective alternatives to contact precautions. Thus, some institutional leaders are questioning whether contact precautions for methicillin-resistant $S$. aureus are still of value to their hospital or health care venue. This review examines the literature regarding the use of contact precautions for methicillin-resistant $S$. aureus. The uncertainties about contact precaution measures and alternative approaches to methicillin-resistant $S$. aureus control are highlighted.
\end{abstract}

Keywords Methicillin-resistant Staphylococcus aureus . Contact precautions · Isolation · Hand hygiene .

Chlorhexidine gluconate

\section{J. Brown ( ()}

Division of Infectious Diseases, Department of Internal Medicine, University of California, Davis Medical Center, 4150

V. Street, PSSB-G500, Sacramento, CA 95817, USA

e-mail: jennifer.brown@ucdmc.ucdavis.edu

\section{J. Brown}

Department of Hospital Epidemiology and Infection Prevention, University of California, Davis Medical Center, Sacramento, USA

\section{Introduction}

Methicillin-resistant Staphylococcus aureus (MRSA) is a major cause of health care-associated infections. The first MRSA isolates were reported in the early 1960s, and since then MRSA has become endemic in hospitals throughout the US and around the world [1]. Infections due to this virulent pathogen can be difficult to treat and are a substantial cause of morbidity and mortality. For these reasons, MRSA continues to be a focus of hospital infection prevention efforts.

Guidelines from the US Centers for Disease Control and Prevention and the Healthcare Infection Control Practices Advisory Committee recommend the use of contact precautions to prevent the transmission of MRSA in acutecare facilities [2-4]. Contact precautions require that, if possible, hospitalized patients with MRSA colonization or infection be placed in single or private rooms. When such rooms are not available, cohorting of patients is acceptable, but more than three feet of separation between beds is then recommended to reduce the unintended sharing of items between patients. Whenever feasible, dedicated equipment (e.g., stethoscopes or blood pressure cuffs) should remain in the isolation room and used only for the isolated patient. In addition to performing proper hand hygiene, health care personnel (HCP) must wear gloves and a protective gown upon entry to the room of a MRSA-colonized or MRSAinfected patient; these items should be discarded before exiting the patient's room.

Despite these recommendations, the merits of contact precautions for MRSA are debated [1, 5-9]. This uncertainty results from the lack of conclusive evidence that contact precautions reduce the transmission of MRSA. Also, contact precautions are resource-intensive and may have detrimental effects on patient care and well-being. 
Hence, some institutional leaders are reassessing whether contact precautions for MRSA are of value to their hospital or health care venue. This review examines the literature regarding the use of contact precautions for MRSA. The uncertainties about contact precaution measures and alternative approaches to MRSA control are highlighted.

\section{What is the Rationale for Contact Precautions?}

The rationale for contact precautions arose from insight into the various modes of nosocomial MRSA transmission. Within health care facilities, the spread of MRSA from one patient to another can occur by HCP-patient transmission or via environmental transmission.

\section{HCP-Patient Transmission}

HCP who interact with MRSA-colonized or MRSA-infected patients can become transiently contaminated and thus become vectors for MRSA transmission to other patients $[10 \bullet, 11,12]$. In one prospective cohort study at a tertiary care hospital, Morgan and colleagues found that $14 \%$ of HCP-patient interactions in intensive care units (ICUs) resulted in MRSA-contamination of HCP gloves or gowns [11]. Similar findings were noted in another study in which 17 and $6 \%$ of HCP who cared for patients with MRSA acquired the organism on their gloves or gown, respectively [12]. Factors associated with an increased risk for HCP contamination include time in a patient's room $>5$ min, performing physical examinations, contact with an endotracheal tube or tracheostomy site, and the presence of a percutaneous gastrostomy or jejunostomy tube. Less often, MRSA can be transmitted directly from MRSAcolonized HCPs to patients [8].

\section{Environmental Transmission}

Patients can acquire MRSA also from contaminated surfaces or objects in their health care environment. This can result from direct contact with contaminated objects or from HCP who have touched contaminated items. Several studies have demonstrated that health care environments can easily become reservoirs for MRSA [11, 13-17]. For example, Hardy and colleagues compared environmental specimens obtained from nine ICUs to MRSA isolates obtained from patients who were cared for in those units; MRSA was found in $21.8 \%$ (188/864) of environmental specimens [15]. Notably, the pulsed field gel electrophoresis profiles were indistinguishable 35.7 and $57.1 \%$ of the time when patient isolates were compared to isolates obtained from their immediate care environment or from anywhere in the ICU, respectively. Others have reported that the frequency of hand contamination among $\mathrm{HCP}$ is equivalent whether the contamination occurs from contact with patients or from contact with contaminated surfaces in patient rooms; both are significant routes for MRSA spread [18].

Overall, it is estimated that $20-40 \%$ of hospitalacquired infections result from cross-infection via contaminated HCP or the care environment [16]. Thus, the components of contact precautions are designed to impede these modes of nosocomial transmission.

\section{Do Contact Precautions Reduce MRSA Transmission?}

Many studies have credited the use of contact precautions for decreasing the institutional spread of MRSA. However, whether contact precautions should be recommended for MRSA control remains controversial [1-9].

\section{Limitations Within the Literature}

When appraising the literature on contact precautions, it is important to recognize that certain limitations often are present. First, since health care-associated infections are fairly uncommon, studies often are hampered by insufficient sample sizes and are underpowered for determining the efficacy of contact precautions [1, 7]. Also, many early studies were conducted in outbreak or epidemic settings. As a result, their findings may not be applicable to health care venues in which MRSA is considered endemic [6-8]. Moreover, contact precautions often are evaluated in combination with other measures, such as decolonization; thus it is difficult to differentiate the effects of these measures from those of contact precautions. Finally, the validity of many studies has been criticized for suboptimal or unmonitored HCP compliance with contact precautions $[5,7]$.

Does the Literature Support the Use of Contact Precautions?

In 2004, Cooper and colleagues published their systematic review of the literature regarding the effectiveness of isolation measures in the management of MRSA in hospitals [6]. Among 46 studies, 45 described multiple, simultaneous infection control interventions. As a result, the authors were unable to assess the relative contribution of each of the interventions. And, though 32/46 studies reported evidence for reduced MRSA transmission, in over half the evidence was considered weak. Six interrupted time-series studies had stronger evidence for MRSA control by contact precautions, but most of these studies involved outbreak situations [19-22]. Overall, Cooper et al. concluded that 
major methodological weaknesses exist throughout the body of research and, therefore, the routine use of contact precautions for MRSA control is not supportable. This position notwithstanding, the authors advised that isolation measures, as recommended in national guidelines, be continued pending further research because "the lack of evidence should not be mistaken for evidence of lack of effect". In another extensive literature review, Marshall and colleagues specifically addressed the question of whether contact precautions reduce MRSA transmission in MRSA-endemic settings [1]. Similarly, they did not find strong, methodologically sound evidence to support the use of contact precautions to control MRSA.

Some investigators have attempted to parse out which components of contact precautions are worthwhile vs those that are dispensable. In a prospective interrupted timeseries study in the ICUs of two academic hospitals, Cepeda et al. evaluated whether placing MRSA patients in single/ cohorted rooms reduces MRSA transmission [23]. In this study, MRSA screens were collected at admission and weekly thereafter. For all patients, HCP used disposable aprons (instead of gowns), and gloves were worn only for invasive procedures, washing and turning patients, and contact with mucous membranes or body fluids. In the second six months of the year-long study, MRSA-positive patients were no longer moved to single rooms or cohorted unless they required isolation for other pathogens. Results of a crude (unadjusted) Cox proportional-hazards model of the study showed no evidence of increased MRSA transmission during the non-moving phase (0.73 [95\% CI $0.49-1.10], P=0.94$ one-sided). In another study, Bessesen and colleagues addressed the question of whether the use of gowns is necessary to reduce MRSA transmission [24]. The study took place in two Veterans Health Administration hospitals where a bundle of control measures was implemented. The measures included institutional culture change, surveillance for MRSA infection and transmission, and active screening for colonization. Yet, one hospital employed full contact precautions, whereas the other used modified contact precautions (the use of gloves but not gowns). Over the four-year study period the two hospitals had comparable MRSA transmission rates; thus, the use of gowns did not appear to be beneficial. A fundamental weakness of both studies is that there was an ample window for nosocomial MRSA transmission to occur because the time elapsed from admission to MRSA screening result was about 4-5 days.

Other investigators aimed to resolve the shortcomings of earlier studies through the use of more stringent study designs. Huskins et al. conducted a rigorous, multicenter, cluster-randomized trial in which active, culture-based MRSA surveillance plus expanded contact precautions were employed [25]. The authors found that these measures did not reduce the incidence of MRSA colonization or infection in their adult ICUs. However, akin to prior studies, HCP compliance with gloves, gowns, and hand hygiene was suboptimal and, the turnaround time for reporting MRSA-positive surveillance cultures was 4-6 days. In contrast, Marshall and colleagues used rapid testing methods and multiple-body site MRSA screening to evaluate contact precautions vs their institution's usual practice of plastic aprons for all patient contact, no MRSA screening, and no single room/cohorting of MRSA-infected patients [26]. This prospective, interrupted time series was conducted in a tertiary care center's ICU. Nose, throat, groin, and axilla swabs were collected on admission, twice weekly, and at or within $48 \mathrm{~h}$ of discharge. In the study's intervention phase, all swabs were processed by polymerase chain reaction (PCR) methods, and patients with MRSA-positive results were placed into contact precautions. MRSA acquisition rates (per 1,000 at-risk patient days) were 18.5 for their usual practice versus 7.9 during the intervention phase, with an adjusted hazard ratio $0.39 \quad(95 \%$ CI 0.24-0.62). Overall, this intensified strategy was credited with a $60 \%$ reduction in nosocomial MRSA acquisition. Notably, the median time from swab collection to results was relatively short (18 h). Also, HCP compliance with contact precaution measures was unusually high $(>85 \%)$, owing to the employment of research nurses for the study and possibly a Hawthorne effect. Whether these single-center results are reproducible, practical, or feasible in other health care institutions is unknown.

\section{Do Contact Precautions Impede Patient Care?}

The goal of contact precautions is to protect patients who may be at risk of acquiring nosocomial infections. However, there is growing concern that these infection prevention measures can lead to adverse consequences for patients who are isolated $[5,7,27]$. Systematic literature reviews have identified that contact precautions may negatively impact several aspects of patient care including HCP-patient interactions, patient safety, and patient psychosocial health $[27,28]$. Others have questioned the effects of contact precautions on health care costs and resource utilization $[7,29]$.

\section{HCP-Patient Interactions}

One possible drawback to contact precautions is that the effort required to don and doff gloves and gowns creates a disincentive for HCP to interact with patients [30-32]. In 1999, Kirkland and Weinstein published their study on the influence of contact precautions on HCP-patient interactions in a medical ICU. Fourteen patients in contact precautions and 21 patients not in contact precautions were 
observed. The rate of direct HCP-patient contact was $50 \%$ less for patients in contact precautions as compared to that for non-isolated patients $(P=0.3)$ [30]. In another prospective cohort study, Saint et al. used an embedded observer to record HCP-patient encounters during morning rounds at two academic medical centers [32]. It was noted that attending physicians examined $35 \%(11 / 31)$ of patients who were in contact precautions and $73 \%(79 / 108)$ of nonisolated patients (relative risk, 0.49 [95\% CI 0.30-0.79], $P<.001)$. Similarly, Evans et al. found that HCP-patient interactions were half as numerous (5.3 vs. 10.9 hourly encounters, $P<.001$ ) and HCP-patient contact time was $22 \%$ less (29 vs. $37 \mathrm{~min}$ per h, $P=.008$ ) for surgical patients in contact precautions vs those not in contact precautions [31]. In contrast to these studies, two trials in pediatric patient populations found comparable HCPpatient encounters among isolated and non-isolated children $[33,34]$. In one of these trials, Cohen found that attending physicians spent about nine minutes in the hospital rooms of both isolated $(n=24)$ and non-isolated $(n=41)$ children [33]. Critics of these studies cite small sample sizes, limited details about patient selection, and inadequate controls. As a result, the extent to which contact precautions affect HCPpatient interactions is not well-defined [10••].

\section{Patient Safety and Psychosocial Health}

The safety and psychosocial health of patients who are placed in contact precautions also has been scrutinized [28, 35-37]. One retrospective chart review by Stelfox and colleagues examined the care provided to 150 patients in contact precautions for MRSA and 300 non-isolated controls at two academic medical centers [36]. Compared with controls, isolated patients were more likely to have unrecorded vital signs $(51 \%$ vs. $31 \%, P<.001)$ and days without physician progress notes ( 26 vs. $13 \%, P<.001$ ) or nursing notes $(14$ vs. $10 \%, P<.001)$. Although the mortality rate was similar between the groups, isolated patients were almost seven times more likely to have incurred preventable events, such as falls, pressure ulcers, and fluid or electrolyte disorders. One limitation of the study was that patients in contact precautions had longer hospital stays than those who were not isolated, which may have increased the opportunity for adverse events to occur.

A number of trials have shown that isolated patients experience increased rates of depression and anxiety [28, 37]. For example, Tarzi et al. used the Geriatric Depression Scale-Short Form to assess elderly patients who were hospitalized in rehabilitation wards for at least four weeks; among the 22 patients in contact precautions, $77 \%$ had depression as compared to $33 \%$ of the 20 non-isolated patients $(P<.01)$ [37]. Likewise, anxiety rates were significantly higher in the contact precautions cohort. However, these are small studies and others have shown that patients who are placed in contact precautions have higher rates of pre-hospitalization depression and anxiety [35].

\section{Health Care Costs and Resource Utilization}

As institutional leaders grapple with how best to allocate their resources, they may be faced with deciding whether contact precautions for MRSA are essential for optimal patient care. Alternatively, do these measures unnecessarily divert resources away from other infection prevention or organizational needs? Contact precaution programs accrue considerable costs, including those for gloves, gowns, isolation rooms, screening tests, and personnel. They can also diminish the availability of hospital beds and delay admissions or discharges $[30,36]$. On the other hand, the costs incurred for MRSA infections can be substantial. In 2013, Farbman et al. published their systematic review of 36 studies in which the cost-effectiveness or cost-benefit of MRSA infection control interventions were reported [29]. They found that, among 18 of the studies that itemized savings and costs, the median save:cost ratio was US\$7.16 (IQR 1.37-16) and the median net global saving was US\$23K (IQR 3194-50,049) per month. The majority $(83 \%)$ of the 18 studies showed that infection control interventions were economically justified. Notably, in two studies in which the interventions were not justified, PCR methods were used for patient screening. The results of this analysis should be interpreted cautiously, though, because of the heterogeneous nature of the studies. For example, in more than half of the studies, single room/ cohorting strategies and MRSA screening were not used, and HCP compliance monitoring was poor. Moreover, higher save:cost ratios were observed in hospitals with fewer than 500 beds and higher rates of MRSA-endemicity. Thus, it is difficult to generalize the results of the study to all types of health care settings.

\section{Are Horizontal Approaches to Infection Prevention Better?}

Vertical infection prevention strategies, such as contact precautions for MRSA, are designed to target specific pathogens. In contrast, horizontal infection prevention measures, which are preferred by some experts, combat a broad range of pathogens simultaneously [7, 26, 38•, 39]. Horizontal strategies include population-based approaches and host-based approaches.

Population-Based Infection Prevention Approaches

Examples of population-based strategies are hand hygiene, antibiotic stewardship, care bundles, and environmental 
cleaning $[16,26,38 \bullet]$. A comprehensive discussion of each of these is beyond the scope of this review; however, hand hygiene merits particular attention.

Some authorities argue that adequate hand hygiene, rather than full contact precautions, is sufficient to control the nosocomial spread of MRSA [1, 7, 40]. A number of studies have clearly correlated declines in the rates of MRSA, and other hospital pathogens, with improved hand hygiene practices [3, 10••, 41, 42]. For example, in one academic hospital in which HCP hand hygiene compliance rates increased from 48 to $66 \%$, a significant reduction in nosocomial MRSA transmission from 2.16 to 0.93 episodes per 10,000 patient-days $(P<.001)$ occurred [41]. In addition, the rate of nosocomial infections as a whole declined. Simulation models also have illustrated the correlation between suboptimal hand hygiene and the development of hospital infections [43, 44]. In one elaborate model, Sadsad and colleagues showed that daily prevalence and incidence rates of MRSA decreased exponentially as HCP hand hygiene compliance increased [44]. Moreover, the authors demonstrated that hand hygiene compliance can drive down MRSA rates more successfully than other measures, such as patient isolation, cohorting, or active surveillance. Another model simulated encounters between patients and hand hygiene non-compliant HCP; the investigators calculated that non-compliant HCP who cared for 3,340 MRSA-colonized patients created 980 new MRSA infections among other patients for whom they cared [43]. The estimated mean cost per new infection was US $\$ 53,598$ and the mean cost per noncompliant event was US\$52.53. For a 200-bed hospital, this would amount to an extra US\$1.7 million per year for MRSA-related expenditures. On the other hand, they estimated that a $1 \%$ increase in hand hygiene compliance would save almost US $\$ 40 \mathrm{~K}$ annually.

Despite the evidence that hand hygiene practices can reduce nosocomial infections, $\mathrm{HCP}$ adherence with these practices is notoriously low (mean $40 \%$ ) [40]. Studies that describe successful hand hygiene programs have been published, as have guidelines for hand hygiene in hospitals $[40,45]$.

\section{Host-based approaches}

The aim of host-based approaches, such as the administration of intranasal mupirocin and bathing with chlorhexidine gluconate $(\mathrm{CHG})$, is to reduce the bacterial burden of colonized or infected source patients. In doing so, patients are protected from their own microbiota, and, the opportunities for patient-to-patient transmission of pathogens are reduced [46*0]. Intranasal mupirocin can eradicate both MRSA and methicillin-susceptible $S$. aureus in most nasal carriers [25, 46••, 47]. However the effects of mupirocin can be transient and, this approach does not eradicate bacteria at extra-nasal body sites, such as the throat or perineum. CHG is an antiseptic agent with activity against a broad range of pathogens, including MRSA. It has residual antibacterial activity on the skin after application and a low toxicity profile [48]. Studies have credited CHG bathing for lowering the concentration of bacteria on patients' bodies and reducing the rates of many types of bacterial infections. [46••, 48-51]

In one of the most extensive (43 hospitals, 74 ICUs) and rigorous trials to-date, Huang et al. performed a clusterrandomized, pragmatic, comparative-effectiveness trial in which targeted and universal decolonization were compared with one another, and with MRSA screening and contact precautions alone [46*0]. In one group of patients, MRSA screening was performed and contact precautions were implemented for those with MRSA colonization/ infection (screening and isolation group). Two other groups underwent the same isolation procedures, but in one, MRSA screening plus intranasal mupirocin and CHG bathing for MRSA-positive patients was performed (targeted decolonization group); the other group did not undergo MRSA screening, but all patients received mupirocin and CHG bathing (universal decolonization group). Overall, the universal decolonization strategy was the most effective infection prevention strategy; it significantly reduced MRSA-positive clinical cultures by $37 \%$ and bloodstream infections from any pathogen by $44 \%$. Another trial by Derde et al. compared universal CHG bathing plus hand hygiene improvement to MRSA (and vancomycin-resistant Enterococcus) screening plus contact precautions [49]. The authors found that improved hand hygiene (77\% compliance), along with CHG bathing, reduced the rates of new acquisitions of antimicrobialresistant bacteria more effectively than did screening plus contact precautions.

The results of these studies suggest that universal decolonization and improved hand hygiene can prevent infections, avert the need for MRSA screening, and decrease contact isolation requirements. However, since both studies were conducted in ICUs with higher-thanaverage hand hygiene compliance rates, the results may not be generalizable to other health care settings. In addition, concerns have been raised that widespread mupirocin or CHG use could promote the emergence of mupirocin- or CHG-resistant bacteria [10••, 48, 52].

\section{Conclusions}

Contact precautions have been promoted as a means of reducing the nosocomial spread of MRSA. However, evidence to support their efficacy for MRSA control is not entirely compelling. Moreover, these measures are 
resource-intensive and may adversely affect patient care. In the present day, contact precautions remain important for outbreak situations or to curb the spread of non-MRSA drug-resistant pathogens. Yet, whether they are necessary for the general constraint of MRSA is unclear. Evidence exists that some horizontal infection prevention strategies may be as effective, but less resource-intensive, than contact precautions for MRSA control. Taken together, these factors may prompt institutions to reassess the value of contact precautions for MRSA. Whether the use of contact precautions is deemed justifiable will depend on each institution's needs and capabilities. For those that elect to use contact precautions for MRSA, it is imperative that these measures do not interfere with patient well-being. On the other hand, institutions that opt for horizontal prevention strategies must commit to achieving and maintaining high rates of compliance for this approach to be successful.

\section{Compliance with Ethics Guidelines}

Conflict of Interest Dr. Jennifer Brown reports no conflicts of interest.

Human and Animal Rights and Informed Consent This article does not contain any studies with human or animal subjects performed by any of the authors.

\section{References}

Papers of particular interest, published recently, have been highlighted as:

- Of importance

-• Of major importance

1. Marshall C, Wesselingh S, McDonald M, et al. Control of endemic MRSA-what is the evidence? A personal view. J Hosp Infect. 2004;56(4):253-68.

2. Siegel JD, Rhinehart E, Jackson M, et al. 2007 Guideline for isolation precautions: preventing transmission of infectious agents in health care settings. Am J Infect Control. 2007;35(10 Suppl 2):S65-164.

3. Siegel JD, Rhinehart E, Jackson M, et al. Management of multidrug-resistant organisms in health care settings, 2006. Am J Infect Control. 2007;35(10 Suppl 2):S165-93.

4. Yokoe DS, Anderson DJ, Berenholtz SM, et al. A compendium of strategies to prevent healthcare-associated infections in acute care hospitals: 2014 updates. Infect Control Hosp Epidemiol. 2014; 35(8):967-77.

5. Aboelela SW, Saiman L, Stone P, et al. Effectiveness of barrier precautions and surveillance cultures to control transmission of multidrug-resistant organisms: a systematic review of the literature. Am J Infect Control. 2006;34(8):484-94.

6. Cooper BS, Stone SP, Kibbler CC, et al. Isolation measures in the hospital management of methicillin resistant Staphylococcus aureus (MRSA): systematic review of the literature. BMJ. 2004;329(7465):533.

7. Kirkland KB. Taking off the gloves: toward a less dogmatic approach to the use of contact isolation. Clin Infect Dis. 2009;48(6):766-71.
8. Landelle C, Pagani L, Harbarth S. Is patient isolation the single most important measure to prevent the spread of multidrugresistant pathogens? Virulence. 2013;4(2):163-71.

9. Tacconelli E. Screening and isolation for infection control. J Hosp Infect. 2009;73(4):371-7.

10. $\bullet$ Calfee DP, Salgado CD, Milstone AM, et al. Strategies to prevent methicillin-resistant Staphylococcus aureus transmission and infection in acute care hospitals: 2014 update. Infect Control Hosp Epidemiol. 2014;35(7):772-96. This expert guidance document is the product of a collaboration between the Society for Healthcare Epidemiology of America (SHEA), the Infectious Diseases Society of America (IDSA), the American Hospital Association (AHA, the Association of Professionals in Infection Prevention and Epidemiology (APIC), and The Joint Commission. It provides updated recommendations for implementing and prioritizing MRSA prevention efforts.

11. Morgan DJ, Rogawski E, Thom KA, et al. Transfer of multidrugresistant bacteria to healthcare workers' gloves and gowns after patient contact increases with environmental contamination. Crit Care Med. 2012;40(4):1045-51.

12. Snyder GM, Thom KA, Furuno JP, et al. Detection of methicillinresistant Staphylococcus aureus and vancomycin-resistant enterococci on the gowns and gloves of healthcare workers. Infect Control Hosp Epidemiol. 2008;29(7):583-9.

13. Boyce JM, Potter-Bynoe G, Chenevert C, et al. Environmental contamination due to methicillin-resistant Staphylococcus aureus: possible infection control implications. Infect Control Hosp Epidemiol. 1997;18(9):622-7.

14. Chang S, Sethi AK, Stiefel U, et al. Occurrence of skin and environmental contamination with methicillin-resistant Staphylococcus aureus before results of polymerase chain reaction at hospital admission become available. Infect Control Hosp Epidemiol. 2010;31(6):607-12.

15. Hardy KJ, Oppenheim BA, Gossain S, et al. A study of the relationship between environmental contamination with methicillinresistant Staphylococcus aureus (MRSA) and patients' acquisition of MRSA. Infect Control Hosp Epidemiol. 2006;27(2):127-32.

16. Weber DJ, Anderson D, Rutala WA. The role of the surface environment in healthcare-associated infections. Curr Opin Infect Dis. 2013;26(4):338-44.

17. Wilson AP, Hayman S, Whitehouse $\mathrm{T}$, et al. Importance of the environment for patient acquisition of methicillin-resistant Staphylococcus aureus in the intensive care unit: a baseline study. Crit Care Med. 2007;35(10):2275-9.

18. Stiefel U, Cadnum JL, Eckstein BC, et al. Contamination of hands with methicillin-resistant Staphylococcus aureus after contact with environmental surfaces and after contact with the skin of colonized patients. Infect Control Hosp Epidemiol. 2011;32(2):185-7.

19. Coello R, Jiménez J, García M, et al. Prospective study of infection, colonization and carriage of methicillin-resistant Staphylococcus aureus in an outbreak affecting 990 patients. Eur J Clin Microbiol Infect Dis. 1994;13(1):74-81.

20. Cosseron-Zerbib M, Roque Afonso AM, Naas T, et al. A control programme for MRSA (methicillin-resistant Staphylococcus aureus) containment in a paediatric intensive care unit: evaluation and impact on infections caused by other micro-organisms. J Hosp Infect. 1998;40(3):225-35.

21. Duckworth GJ, Lothian JL, Williams JD. Methicillin-resistant Staphylococcus aureus: report of an outbreak in a London teaching hospital. J Hosp Infect. 1988;11(1):1-15.

22. Harbarth S, Martin Y, Rohner P, et al. Effect of delayed infection control measures on a hospital outbreak of methicillin-resistant Staphylococcus aureus. J Hosp Infect. 2000;46(1):43-9.

23. Cepeda JA, Whitehouse T, Cooper B, et al. Isolation of patients in single rooms or cohorts to reduce spread of MRSA in 
intensive-care units: prospective two-centre study. Lancet. 2005;365(9456):295-304.

24. Bessesen MT, Lopez K, Guerin K, et al. Comparison of control strategies for methicillin-resistant Staphylococcus aureus. Am J Infect Control. 2013;41(11):1048-52.

25. Huskins WC, Huckabee CM, O'Grady NP, et al. Intervention to reduce transmission of resistant bacteria in intensive care. $\mathrm{N}$ Engl J Med. 2011;364(15):1407-18.

26. Marshall C, Richards M, McBryde E. Do active surveillance and contact precautions reduce MRSA acquisition? A prospective interrupted time series. PLoS ONE. 2013;8(3):e58112.

27. Morgan DJ, Diekema DJ, Sepkowitz K, et al. Adverse outcomes associated with contact precautions: a review of the literature. Am J Infect Control. 2009;37(2):85-93.

28. Abad C, Fearday A, Safdar N. Adverse effects of isolation in hospitalised patients: a systematic review. J Hosp Infect. 2010;76(2):97-102.

29. Farbman L, Avni T, Rubinovitch B, et al. Cost-benefit of infection control interventions targeting methicillin-resistant Staphylococcus aureus in hospitals: systematic review. Clin Microbiol Infect. 2013;19(12):E582-93.

30. Kirkland KB, Weinstein JM. Adverse effects of contact isolation. Lancet. 1999;354(9185):1177-8.

31. Evans HL, Shaffer MM, Hughes MG, et al. Contact isolation in surgical patients: a barrier to care? Surgery. 2003;134(2):180-8.

32. Saint S, Higgins LA, Nallamothu BK, et al. Do physicians examine patients in contact isolation less frequently? A brief report. Am J Infect Control. 2003;31(6):354-6.

33. Cohen E, Austin J, Weinstein M, et al. Care of children isolated for infection control: a prospective observational cohort study. Pediatrics. 2008;122(2):e411-5.

34. Klein BS, Perloff WH, Maki DG. Reduction of nosocomial infection during pediatric intensive care by protective isolation. N Engl J Med. 1989;320(26):1714-21.

35. Day HR, Perencevich EN, Harris AD, et al. Depression, anxiety, and moods of hospitalized patients under contact precautions. Infect Control Hosp Epidemiol. 2013;34(3):251-8.

36. Stelfox HT, Bates DW, Redelmeier DA. Safety of patients isolated for infection control. JAMA. 2003;290(14):1899-905.

37. Tarzi S, Kennedy P, Stone S, et al. Methicillin-resistant Staphylococcus aureus: psychological impact of hospitalization and isolation in an older adult population. J Hosp Infect. 2001;49(4):250-4.

38. - Septimus E, Weinstein RA, Perl TM, et al. Approaches for preventing healthcare-associated infections: go long or go wide? Infect Control Hosp Epidemiol. 2014;35(7):797-801. This commentary discusses vertical vs horizontal infection prevention strategies. The authors also make recommendations for improving the future investigation and management of epidemiologically important pathogens across heterogeneous health care settings.

39. Wenzel RP, Bearman G, Edmond MB. Screening for MRSA: a flawed hospital infection control intervention. Infect Control Hosp Epidemiol. 2008;29(11):1012-8.

40. Boyce JM, Pittet D; Healthcare Infection Control Practices Advisory Committee; HICPAC/SHEA/APIC/IDSA Hand Hygiene Task
Force. Guideline for Hand Hygiene in Health-Care Settings. Recommendations of the Healthcare Infection Control Practices Advisory Committee and the HICPAC/SHEA/APIC/IDSA Hand Hygiene Task Force. Society for Healthcare Epidemiology of America/Association for Professionals in Infection Control/Infectious Diseases Society of America. MMWR Recomm Rep. 2002;51(RR-16):1-45, quiz CE1-4.

41. Pittet D, Hugonnet S, Harbarth S, et al. Effectiveness of a hospital-wide programme to improve compliance with hand hygiene. Infection Control Programme. Lancet. 2000;356(9238):1307-12.

42. Sroka S, Gastmeier P, Meyer E. Impact of alcohol hand-rub use on meticillin-resistant Staphylococcus aureus: an analysis of the literature. J Hosp Infect. 2010;74(3):204-11.

43. Cummings KL, Anderson DJ, Kaye KS. Hand hygiene noncompliance and the cost of hospital-acquired methicillin-resistant Staphylococcus aureus infection. Infect Control Hosp Epidemiol. 2010;31(4):357-64.

44. Sadsad R, Sintchenko V, McDonnell GD, et al. Effectiveness of hospital-wide methicillin-resistant Staphylococcus aureus (MRSA) infection control policies differs by ward specialty. PLoS ONE. 2013;8(12):e83099.

45. Sakamoto F, Yamada H, Suzuki C, et al. Increased use of alcohol-based hand sanitizers and successful eradication of methicillin-resistant Staphylococcus aureus from a neonatal intensive care unit: a multivariate time series analysis. Am J Infect Control. 2010;38(7):529-34.

46. • Huang SS, Septimus E, Kleinman K, et al. Targeted versus universal decolonization to prevent ICU infection. N Engl J Med. 2013;368(24):2255-65. This robust study demonstrates that universal decolonization strategies are more effective for MRSA control than screening and isolation strategies.

47. Ammerlaan HS, Kluytmans JA, Wertheim HF, et al. Eradication of methicillin-resistant Staphylococcus aureus carriage: a systematic review. Clin Infect Dis. 2009;48(7):922-30.

48. Climo MW, Yokoe DS, Warren DK, et al. Effect of daily chlorhexidine bathing on hospital-acquired infection. N Engl J Med. 2013;368(6):533-42.

49. Derde LP, Cooper BS, Goossens H, et al. Interventions to reduce colonisation and transmission of antimicrobial-resistant bacteria in intensive care units: an interrupted time series study and cluster randomised trial. Lancet Infect Dis. 2014;14(1):31-9.

50. Popovich KJ, Hota B, Hayes R, et al. Effectiveness of routine patient cleansing with chlorhexidine gluconate for infection prevention in the medical intensive care unit. Infect Control Hosp Epidemiol. 2009;30(10):959-63.

51. Vernon MO, Hayden MK, Trick WE, et al. Chlorhexidine gluconate to cleanse patients in a medical intensive care unit: the effectiveness of source control to reduce the bioburden of vancomycin-resistant enterococci. Arch Intern Med. 2006;166(3):306-12.

52. Edgeworth JD. Has decolonization played a central role in the decline in UK methicillin-resistant Staphylococcus aureus transmission? A focus on evidence from intensive care. J Antimicrob Chemother. 2011;66(Suppl 2):ii41-7. 